\title{
EL DEBATE INTERNACIONAL SOBRE EL DESARROLLO SOSTENIBLE
}

\author{
Eguzki Urteaga \\ Departamento de Sociología \\ Universidad del País Vasco
}

\section{RESUMEN}

El desarrollo sostenible es un concepto político forjado al finalizar los «treinta gloriosos», es decir después de un largo periodo de desarrollo socioeconómico (1945-1975) sinónimo de alto crecimiento, de pleno empleo y de elevado nivel de vida. Las cuestiones medioambientales aparecen entonces como la otra cara de la moneda. El desarrollo sostenible se impone como el reto fundamental del siglo XXI. Presentado como la solución a todos los problemas a los que se enfrenta la humanidad, el desarrollo sostenible sigue siendo un enigma: ¿Cómo se puede crecer, aumentar el bienestar de la población mundial, luchar contra las desigualdades sociales y salvaguardar la dinámica de la biosfera? ¿Hay que confiar en el capitalismo y en la regulación por los precios o, por el contrario, es preciso imponer nuevas normas para limitar los efectos del capitalismo? Si así es, ¿esta normativa debe ser elaborada por actores públicos o privados? Para contestar a estas preguntas es imprescindible detenerse en la historia del debate internacional sobre la noción de desarrollo sostenible.

Palabras clave: desarrollo sostenible - medioambiente - crecimiento - planeta cumbre.

\section{RESUME}

Le développement durable est un concept politique qui voit le jour au terme des «trentes glorieuses», c'est-à-dire après une longue période d'essor socioéconomique (1945-1975) synonyme de croissance élevée, de plein emploi et de niveau de vie élevé. Les questions environnementales apparaissent dès lors comme l'autre face de la monnaie. Le développement durable s'est imposé comme un enjeu majeur du XXIème siècle. Présenté comme une solution aux problèmes auxquels est confronté l'humanité, le développement durable continue à constituer une énigme: comment est-il possible de croître, d'améliorer le bien être social de la population mondiale, de lutter contre les inégalités sociales tout en sauvegardant la dynamique de la biosphère? Faut-il avoir confiance dans le capitalisme et dans la régulation par les prix ou, au contraire, faut-il imposer des règles pour limiter les effets néfastes du capitalisme? S'il en est ainsi, cette législation doit-elle être élaborée 
par les acteurs publics ou privés? La réponse à ces questions suppose de s'attarder sur le débat international sur la notion de développement durable.

Mots clés: développement durable - environnement - croissance - planète - sommet.

\section{En debate sobre el crecimiento}

El primer informe entregado al Club de Roma titulado The Limits of Growth es publicado en 1972. Meadows y su equipo del Massachussets Institute of Technology privilegian un punto de vista global y sistemático. De hecho, los problemas considerados se extienden al conjunto del planeta e interactúan unos con otros. Según Meadows, «desarrollo y medioambiente deben absolutamente ser tratados como un solo y mismo problema» (Meadows et al, 1972: 295), lo que plantea la necesidad de considerar una problemática mundial inspirada de la ecología global emergente. Este estudio se basa sobre una simulación por ordenador de un modelo relativamente simple construido por Forrester y sus colaboradores a partir de cinco parámetros: población, producción alimenticia, industrialización, contaminación y utilización de recursos naturales no renovables. La dinámica de este ecosistema mundial hace que los fenómenos se fortalezcan y conduzcan a un círculo vicioso: un número cada vez más grande de individuos que consumen y contaminan de manera creciente en un mundo cuyos recursos son limitados. Sea cual sea el escenario elegido, este crecimiento exponencial conduce finalmente al derrumbe del sistema.

Esta conclusión pretende generar un debate: «tenemos el convencimiento de que la toma de conciencia de los límites materiales del medioambiente mundial y de las consecuencias trágicas de una explotación no razonada de los recursos terráqueos es indispensable para la aparición de nuevos modos de pensamiento que conduzcan a una revisión fundamental, a la vez del comportamiento de los seres humanos y, posteriormente, de la estructura de la sociedad actual en su conjunto» (Meadows, 1972: 293-294). Más allá de la voluntad de abrir el debate, los autores son favorables al advenimiento de un estado de equilibrio global y de una sociedad estable, ya que la población y el capital son las únicas entidades que deben ser constantes en un mundo en equilibrio. Todas las actividades humanas que no provocan un consumo desatinado de materias no sustituibles o que no deterioran de manera irreversible el medioambiente podrían desarrollarse indefinidamente. En particular, estas actividades que muchos consideran como las más deseables y satisfactorias: educación, arte, investigación, deportes y relaciones humanas, podrían prosperar». Dicho de otra manera, el desarrollo sigue siendo posible. La estabilidad del sistema global impone niveles de población y de inversión constantes; un desfase de unos quince años entre los movimientos de estabilización de estas dos dimensiones debe permitir mejorar el nivel de vida material a escala mundial. Más allá del eslogan del «crecimiento cero» que ha marcado los espíritus y ha sido objeto de discusiones, incluso en el Club de Roma, se plantea el problema del reparto de las riquezas a nivel mundial. Para ello, el crecimiento debe producirse en los países del Sur, al menos durante cierto tiempo, e interrumpirse en los países del Norte. Se trata de una idea presente en el pensamiento de Bairoch (1971) al fin de salir el Tercer Mundo del callejón sin salida en la que se encuentra.

El éxito editorial del Libro de los límites genera un amplio debate. Las críticas conciernen la modelización propuesta, los resultados del estudio y su socio comanditario. Los economistas neoclásicos subrayan que el modelo de Forrester y Meadows no se refiere a la función de producción y al mecanismo de regulación por los precios. Denuncian 
también la ausencia de datos empíricos que permitirían dar crédito a sus predicciones. Creado en 1968 por el industrial Peccei y el director de asuntos científicos de la OCDE, King, el Club de Roma, un grupo de expertos constituido por un centenar de miembros cooptados, provenientes de los sectores empresariales, de la ciencia y de la diplomacia, es acusado de promover una ecología tecnocrática (Braillard, 1982). La polémica crece durante los primeros meses de 1972 mientras que las principales conclusiones del informe Meadows son recogidos en el libro The Ecologist. El objetivo del crecimiento continuo e ilimitado es cuestionado por sus impactos medioambientales y sociales (se subrayan la disminución de la diversidad biológica y la persistencia del desempleo masivo) y proponen sustituirlo por una sociedad estable a largo plazo capaz de perpetuarse para la satisfacción de todos. Numerosos personalidades científicas apoyan este Plan para la supervivencia.

Asimismo, en febrero de 1972, Mansholt, vicepresidente de la Comisión europea, envía una carta al presidente de esta institución en la que expresa su deseo de que Europa lleve a cabo un plan económico que prevé, entre otros aspectos, «la fuerte reducción del consumo de los bienes materiales por habitante, compensada por la extensión de los bienes de equipamiento, la lucha contra la contaminación y el agotamiento de las materias primas» (Reichenbach y Urfer, 1974: 65). Mansholt propone que la Comisión apruebe medidas fiscales así como un sistema de certificados de producción para hacer respetar las normas de limpieza y de reciclaje. Pide también que la investigación sea orientada hacia la utilidad más que hacia el crecimiento. La oposición es inmediata. En Francia, el representante de la patronal CNPF declara que un fuerte crecimiento es indispensable. Por su lado, el secretario general del Partido Comunista galo denuncia el «programa monstruoso» de los dirigentes de la CEE. El debilitamiento del crecimiento y el incremento tanto de la inflación como del paro acabarán enterrando este informe.

La reflexión del Club de Roma sobre los límites del crecimiento continúa a pesar de todo, con la publicación de un segundo informe solicitado a Mesarovic y Pestel (1974) en el que presentan una desagregación del modelo global inicial en diez subsistemas regionales. Después de haber predicho el advenimiento de catástrofes regionales, los autores oponen un crecimiento indiferenciado y únicamente cuantitativo a un crecimiento orgánico y cualitativo. Se trata ante todo de «definir el desarrollo razonable para tal región, teniendo en cuenta los recursos disponibles para la población, de sus coacciones y de sus interdependencias de cualquier naturaleza». La expresión desarrollo sostenible solo aparece bajo la pluma de su presidente tras la lectura de la Estrategia mundial de la conservación de la UICN (1980) (Peccei, 1981: 25).

\section{La conferencia de Estocolmo}

En junio de 1972 tiene lugar en Estocolmo la primera conferencia de las Naciones Unidas sobre el ser humano y su entorno. Su eslogan oficial es «Una sola tierra». En ella se cuestiona la vulnerabilidad del planeta y la interdependencia de los fenómenos que tienen lugar. Se trata de cuidar un «pequeño planeta». En esta época marcada por las imágenes de los primeros pasos del hombre en la luna, Ward y Dubos utilizan la metáfora de la nave espacial tierra para poner de manifiesto, por una parte, el desequilibrio entre la dinámica de la tecnosfera, entendida como el sistema mundial de innovaciones técnicas, inversiones e intercambios comerciales, y la de la biosfera y, por otra parte, el reparto no equitativo de la prosperidad. Para hacer frente a estos retos, el objetivo de la conferencia de Estocolmo es «definir los modelos de comportamiento colectivo que permiten a las civilizaciones continuar a existir». Se trata de «elaborar una política co- 
mún a fin de crear un orden viable» que no se conforme con «ofrecer a las generaciones futuras una mezcla de expansión científica, codicia económica y arrogancia nacional que se observa actualmente».

A pesar de este llamamiento, no se produce una unidad política del planeta, en un contexto marcado por la guerra de Vietnam. Durante su discurso de inauguración, Olof Palme critica, sin citarlo, a Estados Unidos, movilizando a la ecología eco-sistémica y provocando un «ecocidio» del que se observan todavía las consecuencias. Esta conferencia, que plantea la cuestión del enfrentamiento entre desarrollo y medioambiente, asiste a una oposición entre bloques de países: entre el Norte y el Sur pero también entre el Este y el Oeste, con la ausencia de la Unión Soviética y de algunos países del bloque comunista por la falta de reconocimiento de la Alemania del Este. China está presente pero apenas participa en los debates. La prioridad de los países del Tercer Mundo es promocionar su desarrollo y los eslóganes tales como «Nuestra contaminación es la miseria» marcan los espíritus. Esta idea está presente en el cuarto punto del preámbulo de la Declaración de Estocolmo. «En los países en vía de desarrollo, la mayoría de los problemas de medioambientales están causados por el subdesarrollo. En consecuencia, los países en vía de desarrollo deben orientar sus esfuerzos hacia el desarrollo, teniendo en cuenta sus prioridades y la necesidad de preservar y mejorar el medioambiente».

Por su lado, las políticas medioambientales de la mayoría de los países industrializados son recientes. Si aspiran a una cooperación superior en la materia, no prevén integrarlas a escala supranacional. Además de la declaración final, un cierto número de resoluciones es aprobado entre las cuales una de ellas se refiere a las pruebas nucleares. Se toma la decisión de crear un órgano específico en el seno de la ONU encargado de las cuestiones de medioambiente. El Programa de las Naciones Unidas para el medioambiente (PNUE) nace y su sede se ubica en Nairobi, con Strong a su cabeza. Durante este periodo, se produce una importante movilización de las ONG.

\section{El ecodesarrollo}

Un año después de esta conferencia, Strong lanza el término de ecodesarrollo a fin de intentar conciliar puntos de vista opuestos. La expresión es retomada y profundizada en el simposio PNUE/CNUCED que tiene lugar en Cocoyoc (1974). Su declaración final reflexiona sobre los límites internos constituidos por las necesidades humanas y los límites externos representados por los recursos físicos del planeta: «creemos en la posibilidad de establecer nuevos estilos de vida y sistemas más justos, menos arrogantes en sus exigencias materiales, más respetuosos del medioambiente y del planeta en su conjunto. La vía no pasa ni por la espera desesperada de un desastre, ni por la creencia optimista en una sucesión de proezas técnicas. Pasa por una evaluación atenta y relajada de los límites externos, por una búsqueda colectiva de la manera de respetar los límites internos de los derechos fundamentales del ser humano. Pasa por la edificación de estructuras sociales que permitan la expresión de estos derechos y por un paciente trabajo de invención técnica y de modos de desarrollo que valorizan y protegen el patrimonio planetario» (UNEP, 1981: 119). Esta noción, concebida inicialmente para responder a la dinámica de las economías rurales del Tercer Mundo, se ha extendido poco a poco para convertirse en una filosofía general del desarrollo. Se trata de una vía mediana situada a equidistancia de las propuestas extremas de Meadows y de las de los defensores de «la abundancia ilimitada de la naturaleza». Con el transcurso del tiempo, las tres dimensiones del ecodesarrollo (autonomía de las decisiones, satisfacción equitativa de las necesidades y prudencia ecológica) se derivan en otras tantas dimensiones de la sostenibilidad. 
La PNUE convierte el ecodesarrollo en uno de los ejes privilegiados de su estrategia hasta el inicio de los años 1980. El equipo del Centro nacional de investigación sobre el medioambiente y el desarrollo (CIRED) participa en este proyecto a través de un conjunto de contribuciones que serán publicadas posteriormente. El ecodesarrollo ocupa igualmente un lugar central en el tercer informe coordinado y remitido al Club de Roma coordinado por Tinbergen (1976), un especialista en cuestiones de desarrollo que ha recibido el primer Premio de ciencias económicas en memoria de Alfred Nobel. Este documento sobre las relaciones Norte/Sur integra a las propuestas de la Asamblea de las Naciones Unidas que, en 1974, ha hecho un llamamiento a la instauración de un nuevo orden económico internacional más humano y equitativo que desemboque sobre una democracia socioeconómica planetaria. Esta iniciativa, que aspira a elaborar un tratado-marco que fije las reglas fundamentales en materia de cooperación internacional, se produce en un momento en el cual acontecen revuelos geoestratégicos sin precedentes desde el final de la Segunda Guerra mundial.

El tercer informe del Club de Roma se refiere a ello. El crecimiento económico se debilita y el sistema financiero internacional construido en Bretton Woods se derrumba en 1971. A pesar de ello, las dependencias económicas del Tercer Mundo hacia el Norte no se reducen y unas notables desigualdades de riqueza hacen temer el desencadenamiento de la violencia. Desde este punto de vista, la derrota de los Estados Unidos en Vietnam produce un terremoto. En esta atmósfera de guerra, a la vez caliente y fría, la carrera al armamento se intensifica y acapara importantes recursos técnicos y financieros. Creada una década antes, la OPEP conoce un nuevo impulso en 1973 que se traduce por la nacionalización de las empresas petroleras y el aumento del precio del petróleo. Las cuestiones de acceso a las reservas energéticas y materiales empiezan a ser preocupantes. Aparece claramente a través de las discusiones sobre la explotación de ciertos elementos considerados como «patrimonios comunes de la humanidad».

El informe dirigido por Tinberger avanza la idea según la cual los recursos naturales, técnicos y científicos podrían ser considerados como tales: los Estados abandonarían sus competencias sobre los recursos que serían gestionados eficazmente y equitativamente por las instituciones internacionales para el bienestar de la humanidad. A la espera de que semejante «soberanía planetaria descentralizada» se ponga en marcha, las estrategias de desarrollo de los Estados Unidos deben centrarse en la satisfacción de las necesidades básicas. Lo que conduce los autores del informe a subrayar la necesidad de acelerar el crecimiento mundial y de liberalizar el comercio internacional. Además, se produce la marginación progresiva de la noción de ecodesarrollo a favor de la de desarrollo sostenible. La reacción de Kissinger y de la diplomacia norte-americana a la resolución de Cocoyoc ha jugado un papel relevante en esta sustitución semántica (Sachs, 1994: 261). El desarrollo es concebido como un fenómeno de recuperación con respecto al modelo que encarnan las economías occidentales.

\section{La estrategia de la conservación}

El desarrollo sostenible aparece también en un periodo en el cual la ciencia ecológica no se conforma únicamente con describir la amplitud de la crisis medioambiental sino que favorece la toma de decisión para responder a este reto. Esta expresión traduce el reposicionamiento, en el seno de las organizaciones internacionales, del mundo de la conservación de la naturaleza que debe hacer frente a una oposición creciente. Es cierto que su instrumento favorito es el área natural protegido que, en la continuidad de las prácticas coloniales, se basa, por una parte, en una exclusión de las poblaciones locales, acusadas 
de tener prácticas destructivas del medioambiente y, por otra parte, en una inclusión en los intercambios internacionales a través de la actividad turística. El mundo de la conservación promueve una «tercera fase de las políticas de conservación» (Rodary y Castellanet, 2003: 24) caracterizada por la conservación integrada que se traduce por una referencia a la participación de las políticas locales. Se puede resumir en una ecuación: conservación + participación $=$ desarrollo sostenible.

Otros términos atestiguan de este vuelco. El World Wildlife Fund (WWF) habla de ecodesarrollo sostenible en sus informes anuales de 1978 y 1979 (Chartier, 2004). La Unión Internacional para la conservación de la naturaleza (UICN) conoce una evolución similar cuando Strong llega a encabezar esta organización. La estrategia mundial de la conservación (1980) es el primer documento internacional que se propone «contribuir al advenimiento del desarrollo sostenible». Este informe, que ha recibido el apoyo del PNUE, de la FAO, de la UNESCO y del WWF, expone un programa que aspira a conciliar los objetivos de conservación de la naturaleza y de desarrollo de las sociedades humanas. «La conservación es definida como la gestión de la utilización por el hombre de la biósfera de manera que las generaciones actuales saquen el máximo provecho de los recursos naturales y aseguren su continuidad para poder satisfacer las necesidades y las aspiraciones de las nuevas generaciones».

Se distinguen tres objetivos principales: el mantenimiento de los sistemas y procesos ecológicos esenciales para la vida (reciclaje de los alimentos, depuración natural de las aguas, etc), la utilización duradera de las especies y de los ecosistemas, la preservación de la diversidad biológica. Haciendo eco a un debate antiguo que agita el mundo de la protección de la naturaleza, las razones invocadas son tanto morales como económicas: los redactores del informe de la IUCN han comprendido que, gracias al desarrollo de las biotecnologías, los recursos genéticos van a representar unas importantes fuentes de innovación y de beneficio. Puesto que los bosques tropicales concentran une proporción muy importante de estos recursos, su conservación puede ser integrada en la problemática del desarrollo de los países del Sur. Las perspectivas de incremento del comercio internacional de los genes se basan en la firma de contratos de bioprospección que conducen la UICN a inscribir su estrategia en la óptica del NOEI. Este texto constituye un esbozo del futuro convenio sobre la diversidad biológica ratificado en Río.

En 1986, durante la conferencia mundial sobre la conservación y el desarrollo organizada por la UICN en Ottawa, se habla del desarrollo sostenible. Un año antes de la conferencia de Río, la UICN (1991) publica una nueva versión de La estrategia mundial conservando la misma argumentación, es decir integrando las cuestiones de desarrollo y de conservación a fin de mejorar las condiciones de vida de los seres humanos, sin superar la capacidad de carga de los ecosistemas y preservando la diversidad de la vida. Se dice claramente que la noción de desarrollo sostenible es contradictoria en los términos. El objetivo es construir una sociedad duradera y los principios generales deben ser derivados en función de las necesidades y de las concepciones de las diversas comunidades humanas. La transición hacia un modo de vida sostenible pasa, según los autores del informe, por una interrupción del crecimiento demográfico y una disminución de los niveles de consumo de los recursos naturales por parte de las poblaciones más favorecidas del planeta. Para modificar los comportamientos, frente a los instrumentos económicos y jurídicos a poner en marcha, la UICN insiste en la idea de una «ética de la vida sostenible» que se sustenta en la concienciación de que la humanidad pertenece, con las demás especies, a una comunidad de vida. 


\section{El informe Brundtland}

En 1983, la Asamblea general de las Naciones Unidas decide crear la Comisión mundial sobre el medioambiente y el desarrollo (CMED). Se trata de un grupo de trabajo compuesto por miembros del personal político de los diferentes países que se encuentran bajo la presidencia de Brundtland, entonces Primer ministro noruego. El mandato de la CMED es triple: establecer un diagnóstico en materia de problemas medioambientales y de desarrollo y hacer propuestas para una acción innovadora, concreta y realista; considerar nuevas modalidades de cooperación internacional susceptibles de fortalecerla y provocar cambios deseados; aspirar a la toma de conciencia y a la movilización del conjunto de los actores concernidos. Después de cinco años de trabajo, el CMED (1987) publica Nuestro futuro común que consiste en un informe cuyo contenido se refiere a muchas propuestas. "Solo tenemos a una sola y única biósfera para hacernos vivir», subrayan los autores del informe (CMED, 1987: 31). Las cuestiones medioambientales y de desarrollo son considerados conjuntamente: algunos modos de desarrollo deterioran el medioambiente y, por el contrario, un medioambiente deteriorado puede constituir un obstáculo para el desarrollo.

Por lo tanto, no hay una sola crisis, ya que los diferentes ámbitos considerados (población, seguridad alimenticia, erosión de la biodiversidad, energía, contaminación) están vinculados unos con otros. Lo que conduce a encontrar una solución que no es otra que la instauración de un desarrollo sostenible. En esta perspectiva, «el desarrollo sostenible no es un estado de equilibrio sino un proceso de cambio en el cual la explotación de los recursos, la elección de las inversiones, la orientación del desarrollo técnico así como el cambio institucional están determinados en función de las necesidades tanto actuales como futuras» (CMED, 1987: 10-11). Se pone el énfasis sobre la duración del desarrollo antes de tomar en consideración la equidad social que conviene establecer entre las generaciones y en el seno de cada generación. Una última dimensión es el respeto de los sistemas naturales que nos hacen vivir. Constituyen los tres pilares del desarrollo sostenible.

La CMED aspira a una nueva era de crecimiento para responder a las necesidades de la humanidad, avanzando objetivos anuales cifrados del 5\% al 6\% para los países en vía de desarrollo y del 3\% al $4 \%$ para los países industrializados. La calidad de este crecimiento debe cambiar respetando «la no explotación del prójimo» y utilizando técnicas menos consumidoras en energía y en materia. Las administraciones públicas y la industria han integrado el medioambiente en sus decisiones. Es importante que este crecimiento esté al servicio de una concepción amplia del desarrollo, integrando las necesidades fundamentales tales como la alimentación, la energía o el empleo. Este objetivo debe ser diferenciado según los países, puesto que conocen una variedad de condiciones ecológicas y de sistemas económicos y sociales. Si algunas poblaciones deben adaptar su estilo de vida para que sea más respetuoso del medioambiente, otros deben reducir su crecimiento demográfico. La UICN propone la puesta en marcha de estrategias nacionales de conservación susceptibles de aproximarse a los objetivos de conservación y de desarrollo, guardando en la mente que la protección de la naturaleza conlleva también una obligación moral hacia los seres humanos y las generaciones futuras. La necesidad de recurrir al multilateralismo para resolver los problemas internacionales y de instaurar un NOEI está siempre en el orden del día. A este respeto, se hace igualmente referencia a la gestión del patrimonio común. Asimismo, figuran recomendaciones sobre las reformas institucionales y jurídicas que es preciso poner en marcha, entre otros aspectos, a escala internacional.

Más allá de este informe, varios acontecimientos marcan el final de los años 1980. Se puede evocar la caída del Muro de Berlín que conduce a un cambio geopolítico. Brundtland pone el énfasis sobre la importancia de las cuestiones de seguridad en la instauración 
de la problemática del desarrollo sostenible, insistiendo sobre la continuidad entre los trabajos de la Comisión Brandt y Palme de los años 1980 y el informe de la CMED. Si se produce una ligera distensión, en el ámbito militar, el accidente del reactor nuclear de Tchernobyl en abril de 1986 replantea el problema del nuclear civil. La firma, en 1987, después de varios años de negociación, del Protocolo de Montreal sobre las sustancias incriminadas en la disminución de la capa de ozono aparece a muchos observadores como un primer paso en el camino hacia unas negociaciones que conciernen el cambio climático. Si los años 1970 han estado marcados por el miedo y el agotamiento de los recursos naturales, las dudas a propósito de los límites de las capacidades de depuración de la biósfera aumentan desde la mitad de los años 1980, al nivel de las desigualdades económicas, las diferencias se incrementan. Afectados por la caída del precio de las materias primas y de los productos básicos, el encarecimiento del precio del petróleo y la modificación de la política financiera de algunos países ricos que disminuyen la ayuda pública al desarrollo, la mayoría de los países del Tercer Mundo asisten a un aumento de su deuda y se hunden en la crisis.

Veinte años después de la publicación de The Limits of Growth, Meadows y su equipo (1992) publican un nuevo informe. Si las principales conclusiones son similares, algunas de ellas varían. Realiza una distinción entre el crecimiento y el desarrollo puesto que, si en un mundo dado, existen límites al crecimiento, no sucede lo mismo con el desarrollo. A partir de una versión modificada del modelo utilizado en 1972, los autores realizan un conjunto de proyecciones. Teniendo en cuenta el consumo de los recursos, la contaminación emitida y la evolución del sistema actual, que reviste un carácter exponencial, este modelo no es sostenible. Algunos límites ya han sido superados como lo demuestra la disminución de la capa de ozono. Otras señales deben ser interpretadas correctamente si se quiere evitar el derrumbe del sistema. No obstante, una sociedad sostenible es concebible en la medida en que se basa en la técnica, el mercado, las políticas que gestionan los bienes comunes globales y la adopción de una ideología de la suficiencia. Con el transcurso del tiempo, las recomendaciones del Club de Roma hacen hincapie cada vez más en las posibilidades ofrecidas por el progreso técnico, con la idea de reducir la cantidad de materia y de energía necesaria para producir los bienes y servicios (Weizsäcker, 1997).

\section{La Cumbre de la Tierra de Río}

Sugerida por los redactores del informe Brundtland, la conferencia de las Naciones Unidas sobre el medioambiente y el desarrollo tiene lugar en Río de Janeiro del 3 al 14 de junio de 1992, es decir casi veinte años después de la Conferencia de Estocolmo. Por su amplitud (40 000 personas, 108 jefes de Estado y de gobierno, 172 Estados representados), la conferencia de Río es, por aquel entonces, la reunión más importante organizada jamás por la ONU. Se asiste al verdadero lanzamiento mediático de la noción de desarrollo sostenible. Paralelamente a la conferencia interestatal oficial, un global forum tiene lugar, prefigurando los futuros foros sociales donde las ONG alternan entre actividades festivas, manifestaciones políticas y simposios. El mundo empresarial está igualmente presente en este foro alternativo. El secretario general de la cumbre oficial, Strong, que ha realizado una parte de su carrera profesional en la industria petrolífera, ha insistido para que las empresas expresen su punto de vista sobre el desarrollo sostenible. Ha convertido el empresario Schideheyny en su asesor para las cuestiones relativas a la industria y le ha incitado a crear la Business Council for Sustainable Developement (BCSD) con una cincuentena de empresarios. La publicación del libro titulado Cambiar de dirección durante la Cumbre es el mejor ejemplo de la literatura managerial sobre este tema. 
Al término de diez días de discusiones entre los gobiernos, varios textos son aprobados. La Declaración de Río recoge, en preámbulo, la de Estocolmo y pretende darle una continuidad. La comparación de los dos textos es interesante puesto que la cuestión demográfica aparece como menos preocupante, las referencias a la naturaleza y al agotamiento casi han desaparecido así como la utilización de la planificación para conciliar varios objetivos. Por el contrario, el artículo 15 de la Declaración de Río se refiere al principio de precaución. Un plan de acción bautizado Agenda 21 recoge un centenar de acciones a implementar para que el desarrollo sostenible se convierta en una realidad en el siglo XXI. El coste de su aplicación valorado en 600 billones de dólares al horizonte 2000, lo que corresponde al gasto de armamento mundial. Un cierto número de compromisos internacionales han sido tomados: dos convenios-marco han sido firmados, el primero relativo al cambio climático y el segundo a la erosión de la diversidad biológica. Las negociaciones son lanzadas de cara a un tercer convenio que pretende luchar contra la desertificación que será aprobado en 1994. No hay que olvidar que una de las primeras manifestaciones de los problemas globales es la crisis del Sahel que ha empezado en los años 1970 (Raynaut, 1997). Por último, se puede mencionar una declaración de principio sobre la protección de los bosques, puesto que el proyecto de redacción de un convenio sobre el bosque tropical se ha enfrentado a la oposición de algunos países. Se produce también, en el seno de la ONU, la creación de una Comisión de desarrollo sostenible.

\section{La Cumbre de Johanesburgo}

La Cumbre mundial del desarrollo sostenible es organizada en Johannesburgo del 26 de agosto al 4 de septiembre de 2002. Inicialmente, su orden del día se centra en la concreción de los compromisos tomados diez años antes durante la conferencia de Río. Se trata, además, de insistir sobre el aspecto social de la sostenibilidad y de poner el énfasis sobre la pobreza y la precariedad. Mientras tanto, las crisis financieras y los problemas de seguridad han acaparado la atención. Los observadores están de acuerdo para reconocer la debilidad de los resultados de este «Estocolmo + 30». La declaración final de la Cumbre sólo recoge las declaraciones precedentes. El plan de acción solamente consta de compromisos cifrados relativamente vagos, sabiendo que la mayoría de ellos ya ha sido anunciada en las precedentes cumbres internacionales (Declaración de Doha, Declaración del Milenio). Otra vez, se constata un desfase entre las promesas de ayuda a los países del Sur y la realidad de las ayudas financieras concedidas. Las decisiones tomadas en Monterrey en marzo de 2000, en el marco de la conferencia de la ONU sobre la financiación del desarrollo, no han hecho sino reafirmar un objetivo asignado desde hace mucho tiempo.

La Cumbre de Johannesburgo ha ofrecido la oportunidad de presentar nuevas iniciativas, es decir los acuerdos concluidos entre los gobiernos y los actores privados: empresas, ONG, sindicatos, para apoyar la implementación de la Agenda 21. Así, más de 200 acuerdos han sido anunciados durante la Cumbre. Si algunos saludan el reconocimiento de estos actores, otros se preocupan por los deslizamientos que se han producido, puesto que se trata de medidas dispares basadas en unos compromisos voluntarios, lo más a menudo sin procedimientos de evaluación, y que, en la mayoría de los casos, pueden asimilarse a acciones promocionales. Es cierto que las empresas cobran un protagonismo creciente y se sitúan en la punta de la problemática del desarrollo sostenible. Así, la BCSD, que se ha convertido en la World Business Council for Sustainable Development, es un actor que se preocupa por el desarrollo sostenible y que tiene capacidad para responder a este reto. Sin embargo, se trata de una empresa 
acostumbrada a gestionar a corto o largo plazo y a desarrollar estrategias más allá de las fronteras estatales.

\section{Conclusión}

Recordemos que el desarrollo sostenible es un concepto político forjado al finalizar los «treinta gloriosos», es decir después de un largo periodo de mutación socioeconómica (1945-1975) sinónimo de alto crecimiento, de pleno empleo y de elevado nivel de vida. Las cuestiones del medioambiente y del desarrollo aparecen entonces como la otra cara de la moneda. El desarrollo sostenible se impone como el reto fundamental del siglo XXI. Presentado como la solución a todos los problemas a los que se enfrenta la humanidad, el desarrollo sostenible sigue siendo un enigma: ¿Cómo se puede crecer, aumentar el bienestar de la población mundial, luchar contra las desigualdades sociales y salvaguardar la dinámica de la biosfera? ¿Hay que confiar en el capitalismo y la regulación por los precios o, por el contrario, es preciso imponer nuevas normas para limitar los efectos del capitalismo? Si es así, ¿esta normativa debe ser elaborada por actores públicos o privados? Para contestar a estas preguntas es imprescindible detenerse en la historia del debate internacional sobre la noción de desarrollo sostenible.

De hecho, después de ocho años de negociación, el Protocolo de Kyoto entra en vigor en 2005, aunque no responda al reto del cambio climático. En el momento en el cual el diagnóstico realizado por los expertos de «la evaluación de los ecosistemas del milenio» en materia de destrucción de entornos naturales es alarmante, las negociaciones sobre la biodiversidad marcan el paso. Es cierto que la posición de Estados Unidos, que se ha retirado del Protocolo de Kyoto y no ha ratificado el CDB, fragiliza estas políticas. Estas constataciones, así como la dificultad de definir y de poner en marcha unas políticas idóneas a nivel nacional (Lascoumes, 2002), alimentan el escepticismo hacia el objetivo de desarrollo sostenible. A pesar de estos problemas concretos y de la dificultad para dar una definición adecuada, el desarrollo sostenible sigue estando en el orden del día. Por ejemplo, la UNESCO acaba de lanzar la Declaración de la Década de Naciones Unidas para la educación a fin de alcanzar el desarrollo sostenible (2005-2014).

\section{Bibliografía}

BRAILLARD, Ph. (1982): L'imposture du Club de Rome. PUF. Paris.

CHARTIER, D. (2004): «Aux origines des flous sémantiques du développement durable: une lecture critique de la stratégie mondiale de la conservation de la nature», Ecologie et politique, $\mathrm{n}^{\circ} 29$.

CMED, (1987): Notre avenir à tous. Editions du Fleuve. Montréal.

LASCOUMES, P. (2002): L'Eco-pouvoir. Environnements et politiques. La Découverte. Paris.

MEADOWS, D. H., MEADOWS, D. L., RANDERS, J., BEHRENS, W. W. (1972): Halte à la croissance? Fayard. Paris.

PECCEI, A. (1981): 100 pages pour l'avenir. Economica. Paris.

RAYNAUT, C. (1997): Sahels. Diversité et dynamique des relations sociétés-nature. Karthala. Paris.

REICHENBACH, R., URFER, S. (1974): La croissance zéro. PUF. Paris.

RIST, G. (1996): Le Développement. Histoire d'une croyance occidentale. Presses de Sciences Po. Paris.

RODARY, E., CASTELLANET, C. (2003): «Les trois temps de la conservation», in RODARY, E., et al., Conservation de la nature et développement. L'intégration impossible? GRET/Kaethala, Paris. 
SACHS, I. (1994): «Environnement, développement, marché: pour une économie anthropologique», in WEBER, J., Natures, sciences, sociétés, $\mathrm{n}^{\circ} 3$.

UNEP, (1981): In Defense of the Earth. The Basic Texts on Environnement: Founex, Stockholm, Cocoyoc. UNEP Executive Series 1, Nairobi.

WEIZSÄCKER, E.U. VON, LOVINS, A.B., LOVINS, L.H. (1997): Deux fois plus de bien-être en consommant deux fois moins de ressources, Terres vivantes. 
